Yuyi You, MD, PhD

Elizabeth C. Graham, BA

Ting Shen, MS

Con Yiannikas, FRACP

John Parratt, FRACP

Vivek Gupta, PhD

Joshua Barton, FRACP

Michael Dwyer, PhD

Michael H. Barnett,

$\mathrm{PhD}, \mathrm{FRACP}$

Clare L. Fraser,

FRANZCO

Stuart L. Graham,

$\mathrm{PhD}$, FRANZCO

Alexander Klistorner, $\mathrm{MD}, \mathrm{PhD}$

Correspondence to

Dr. You:

yuyi.you@sydney.edu.au or

Dr. Klistorner:

sasha.klistorner@sydney.edu.au

\section{Progressive inner nuclear layer dysfunction in non-optic neuritis eyes in MS}

\section{OPEN}

\section{ABSTRACT}

Objective: To investigate primary retinal functional changes in non-optic neuritis (ON) eyes of patients with MS by full-field electroretinography (ERG).

Methods: Seventy-seven patients with relapsing-remitting MS with no history of clinical ON in at least 1 eye and 30 healthy controls were recruited in the cohort study. Full-field ERGs were recorded, and retinal optical coherence tomography scans were performed to assess the thicknesses of peripapillary retinal nerve fiber layer (RNFL) and retinal ganglion cell layer-inner plexiform layer (GCL-IPL). Annual MRI scans were also carried out to evaluate the disease activity in the brain. Patients were followed up for 3 years.

Results: At baseline, a delayed b-wave peak time was observed in the cone response $(p<0.001)$, which was associated with the thicknesses of RNFL and GCL-IPL. The peak time of the delayed bwave also correlated with the Expanded Disability Status Scale, T2 lesion volume, and disease duration. During the 3-year follow-up, progressive ERG amplitude reduction was observed (both $a$ - and b-waves, $p<0.05)$. There was a correlation between the b-wave amplitude reduction and longitudinal RNFL loss $(p=0.001)$. However, no correlation was found between longitudinal ERG changes and disease activity in the brain.

Conclusions: This study demonstrated progressive inner nuclear layer dysfunction in MS. The borderline a-wave changes suggested some outer retinal dysfunction as well. The correlation between full-field ERG changes and retinal ganglion cell loss suggested that there might be subclinical retinal pathology in MS affecting both outer and inner retinal layers. Neurol Neuroimmunol Neuroinflamm 2018;5:e427; doi: 10.1212/NXI.0000000000000427

\section{GLOSSARY}

DA = dark adapted; EDSS = Expanded Disability Status Scale; ERG = electroretinography; FLAIR = fluid-attenuated inversion recovery; GCL-IPL = ganglion cell layer-inner plexiform layer; INL = inner nuclear layer; $\mathbf{L A}=$ light adapted; LVV = lateral ventricular volume; OCT = optical coherence tomography; $\mathbf{O N}=$ optic neuritis; $\mathbf{O N L}=$ outer nuclear layer; RGC = retinal ganglion cell; RNFL = retinal nerve fiber layer; RRMS = relapsing-remitting MS .

MS is an autoimmune disease involving demyelination and neurodegeneration in the CNS. Neurodegeneration in MS has been recognized as a primary component of MS pathology. ${ }^{1,2}$ Optic neuritis (ON) is usually the manifest onset of MS, and acute inflammation in the optic nerve can lead to axonal transection and retinal ganglion cell (RGC) loss. ${ }^{3}$ However, it has been established that retinal nerve fiber layer (RNFL) thinning occurs not only in ON eyes but also in non-ON eyes in $\mathrm{MS}^{4}$ and is associated with the risk of disability worsening. ${ }^{5}$ RNFL loss in non$\mathrm{ON}$ eyes could be a result of either retrograde transneuronal degeneration ${ }^{6}$ or primary RGC loss.

However, it is still unknown whether the outer layers of the retina, including the outer and inner nuclear layers (INLs), are also affected in MS. A postmortem study showed significant retinal atrophy in the INL in patients with MS. ${ }^{7}$ Outer retinal degeneration has also been identified

From the Save Sight Institute (Y.Y., E.C.G., C.L.F., A.K.), The University of Sydney; Department of Health and Medical Sciences (Y.Y., T.S., V. G., S.L.G., A.K.), Macquarie University; Department of Neurology (C.Y., J.P.), Royal North Shore Hospital; Brain and Mind Center (J.B., M.H. B.), The University of Sydney; Sydney Neuroimaging Analysis Center (M.H.B., A.K.), New South Wales, Australia; and Buffalo Neuroimaging Analysis Center (M.D.), University at Buffalo, NY.

Funding information and disclosures are provided at the end of the article. Go to Neurology.org/nn for full disclosure forms. The Article Processing Charge was funded by the authors.

This is an open access article distributed under the terms of the Creative Commons Attribution-NonCommercial-NoDerivatives License 4.0 (CC BY-NC-ND), which permits downloading and sharing the work provided it is properly cited. The work cannot be changed in any way or used commercially without permission from the journal. 
and was found to be independent of the optic nerve inflammation in the rat model of myelin oligodendrocyte glycoprotein-induced experimental autoimmune encephalomyelitis. ${ }^{8}$ Although most of the previous optical coherence tomography (OCT) studies failed to reveal any retinal architectural changes in the outer retinal layers, Saidha et al. ${ }^{9}$ reported primary retinal neuropathy detected by OCT scans in a subtype of patients with MS, with predominantly macular thinning and more rapid disability progression. However, another group were not able to discriminate the macular thinning phenotype as a distinct subgroup of patients with MS, ${ }^{10}$ suggesting that the concept of primary retinal pathology in MS is still controversial and requires further investigation. Degeneration of retinal layers in relapsing-remitting, secondary progressive, and primary progressive MS was investigated, and INL thinning was observed only in primary progressive MS. ${ }^{11}$ These findings suggest that there might be some subtle outer retinal pathologic changes that are evident in more advanced stages of the disease.

Full-field electroretinography (ERG) is a widely used clinical test, which provides a measure of the neuroretinal function in vivo. ${ }^{12}$ The cornea-negative a-wave is mainly reflecting the photoreceptor function in the outer nuclear layer (ONL), whereas the positive b-wave is primarily generated by bipolar and Müller cells in the INL. Dark and light adaptation allows separated analysis of the signals from the cone and rod pathways. A limited number of cross-sectional ERG studies have been conducted in patients with MS or $\mathrm{ON}$ and showed inconsistent results. ${ }^{13-17}$ No longitudinal ERG studies in MS have been reported to date. Therefore, in the current study, to determine whether there is primary retinal pathology in MS, we examined full-field ERG changes in non-ON eyes in patients with MS with 3-year follow-up. Corresponding RGC loss and disease activity in the brain during the same period were determined by OCT imaging and MRI scans, respectively.

METHODS Participants and ethics. This study was approved by the Human Research Ethics Committee of the
University of Sydney, and written informed consent was obtained from all participants. The study adheres to the tenets of the Declaration of Helsinki.

Patients with consecutive relapsing-remitting MS (RRMS) with no history of clinical $\mathrm{ON}$ in at least 1 eye were enrolled. For patients without a history of ON, a randomly selected eye was used for analysis. Exclusion criteria included a history of other ocular, neurologic, or systemic diseases that could affect the results, such as retinal disorders or other optic neuropathies, diabetes, or medications known to affect the ERG response. Healthy subjects with similar age distribution and male/female ratio were also recruited as controls to minimize selection bias. The number of cases recruited during the study period determined the sample size. Patients with MS underwent annual full ophthalmic examination and brain MRI scans as well as full-field ERG recording and OCT imaging at baseline and year 3 .

Full-field ERG recording. Full-field ERG recordings were performed as per the ISCEV standard ${ }^{18}$ using an ESPION system (Diagnosys LLC, Lowell, MS) to assess the retinal function in the outer and INLs. Pupils were dilated with tropicamide $1 \%$, and the diameter of the dilated pupils were recorded to ensure equivalence for subsequent visits. For dark-adapted (DA) ERGs, rod and combined rod-cone responses were recorded to white flashes with stimulus strengths of $0.01 \mathrm{~cd} \cdot \mathrm{m}^{-1} \cdot \mathrm{s}^{-2}$ (DA 0.01), 3.0 $\mathrm{cd} \cdot \mathrm{m}^{-1} \cdot$ second $\mathrm{d}^{-2}$ (DA 3.0 and oscillatory potentials), and 12.0 $\mathrm{cd} \cdot \mathrm{m}^{-1} \cdot \mathrm{s}^{-2}$ (DA 12.0), respectively, after a 20 -minute dark adaptation. For light-adapted (LA) ERGs, after patients were exposed to $30 \mathrm{~cd} \cdot \mathrm{m}^{-2}$ background luminance for 10 minutes, single-flash cone response (LA 3.0) and $30 \mathrm{~Hz}$ flicker were recorded accordingly. The a- and b-wave peaks were initially identified automatically by the software and then checked and manually adjusted where necessary, and both peak times and amplitudes were analyzed.

OCT imaging. OCT imaging was performed using a Spectralis spectral domain OCT scanner (Heidelberg Engineering, Heidelberg, Germany). A peripapillary circular scan was performed to assess the global RNFL thickness. Also, a macular radial starlike scan was obtained for thickness measurement of ganglion cell layer-inner plexiform layer (GCL-IPL) as previously described. ${ }^{19}$

MRI protocol, lesion identification, and brain atrophy analysis. The MRI protocol used in this study was similar to that which we have used previously. ${ }^{6}$ Briefly, all the study participants underwent annual MRI brain scans at the Brain and Mind Centre, Sydney University on an MR750 3.0-T scanner with an 8-channel head-coil (GE Medical Systems, Milwaukee, WI). Sagittal 3D T1-weighted imaging (GE BRAVO sequence) was acquired with the following image parameters: field of view $($ FOV $)=256 \mathrm{~mm}^{2}$, echo time $(\mathrm{TE})=2.7 \mathrm{~ms}$, repetition time $(\mathrm{TR})=7.2 \mathrm{~ms}$, pixel spacing $=1 \mathrm{~mm}$, flip angle $=12^{\circ}$, and acquisition matrix (Freq. $\times$ Phase $)=256 \times 256$. Axial fluidattenuated inversion recovery sequence (GE CUBE T2 FLAIR) was obtained using the following parameters: $\mathrm{FOV}=240 \mathrm{~mm}^{2}$, acquisition matrix $=256 \times 244, \mathrm{TE}=163 \mathrm{~ms}, \mathrm{TR}=8,000$ $\mathrm{ms}$, flip angle $=90^{\circ}$, and pixel spacing $=0.47 \mathrm{~mm}$.

The MS lesions were identified on coregistered FLAIR T2 and T1 images and segmented semiautomatically using ITKSNAP software (ITK-SNAP is a part of mrDiffusion package available for download at http://www.itksnap.org/pmwiki/ pmwiki.php). Brain atrophy was assessed by lateral ventricular volume (LVV) analysis with the NeuroSTREAM technique as described previously. ${ }^{20}$ T2-FLAIR images were preprocessed and then nonlinearly aligned to 3 previously derived multisubject, 
multiscanner atlases. The inverse transforms were then used to bring standard space ventricular masks into the subject space and to combine them via a locally optimized joint-label fusion scheme. The fused mask was then used to initialize and guide a tuned level set algorithm incorporating a supersampling-based partial volume estimation.

Statistical analysis. Full-field ERG parameters, including amplitudes and peak times of DA rod response, mixed rod/cone response at 2 levels of flash intensity, LA cone response, and 30 $\mathrm{Hz}$ flicker, were compared between patients with MS and controls using the unpaired $t$ test (Graphpad Prism, version 6.0; Graphpad, La Jolla, CA). ERGs at year 3 were compared with baseline ERGs using the paired $t$ test. The correlations between ERG and OCT measurements as well as between ERG parameters and disease severity (Expanded Disability Status Scale [EDSS], MRI lesions, and brain atrophy) were further examined using both simple correlation and multiple regression analysis with least squares (Pearson correlation). The D'Agostino-Pearson omnibus normality test was used to determine whether data were sampled from Gaussian distributions. $p<0.05$ was considered statistically significant.

RESULTS Seventy-seven patients with RRMS and 30 controls were recruited. Demographics of all the participants are shown in table 1.

Baseline study. Functional changes in the INL and $O N L$ in non-ON eyes in MS. A comparison of baseline full-field ERG parameters in non-ON eyes was done between patients with MS and controls. There was a mild delay in the a-wave peak time in DA 3.0 combined rod-cone response in MS ( $p=0.04$, unpaired $t$ test). A more significant delay was observed in the b-wave peak time in LA 3.0 ERG $(p<0.001$, unpaired $t$ test; figure 1A). No difference was observed in ERG amplitudes between MS and controls. All the measurements of ERG peak times and amplitudes are shown in table e-1 at http://links.lww.com/NXI/A20. Male patients showed a smaller b-wave amplitude than females in DA 0.01 ERG $(p=0.03)$. For the patients

\begin{tabular}{|c|c|c|c|}
\hline \multirow[t]{2}{*}{ Table 1} & and clinical characteristics 0 & participants at $b$ & aseline \\
\hline & Patients with RRMS ( $n=77$ ) & Controls $(n=30)$ & $p$ Value \\
\hline Sex & & & $0.63^{a}$ \\
\hline Male & 26 & 8 & \\
\hline Female & 51 & 22 & \\
\hline Age, $y$, mean \pm SEM & $41.25 \pm 1.27$ & $40.27 \pm 2.56$ & $0.71^{b}$ \\
\hline BCVA, LogMAR, mean \pm SEM & $-0.055 \pm 0.015$ & $-0.072 \pm 0.016$ & $0.51^{b}$ \\
\hline $\begin{array}{l}\text { Disease duration, y, median } \\
\text { (range) }\end{array}$ & $4(1-23)$ & - & - \\
\hline EDSS score, median (range) & $1.0(0-6.0)$ & - & - \\
\hline $\begin{array}{l}\text { History of unilateral optic } \\
\text { neuritis }\end{array}$ & 31 & - & - \\
\hline
\end{tabular}

Abbreviations: BCVA = best-corrected visual acuity; EDSS = Expanded Disability Status Scale; LogMAR = logarithm of the minimum angle of resolution; RRMS = relapsing-remitting MS; SEM = standard error of the mean.

${ }^{a}$ Chi squared test.

${ }^{\mathrm{b}}$ Unpaired $t$ test. with a history of unilateral $\mathrm{ON}(\mathrm{n}=31)$, there was a reduction in RNFL in the ON eyes $(91.41 \pm 10$. $89 \mu \mathrm{m}$ for non-ON vs $74.94 \pm 11.93 \mu \mathrm{m}$ for ON; $p$ $<0.0001$, paired $t$ test), but no difference was found in the ERGs between ON and non-ON eyes by intrasubject comparison $(p>0.05$ for all amplitude and peak time parameters; paired $t$-test), which suggested that the ERG functional changes in the outer retinal layers were not related to retrograde transneuronal degeneration.

The INL and ONL function was associated with RNFL/ GCL thicknesses. In previous MS studies, RGC and RNFL loss in non-ON eyes in MS has been well documented. ${ }^{19}$ Here, we further investigated the correlation between the above delayed ERG peak times and OCT measurements including both global RNFL and macula GCL-IPL thicknesses. The a-wave peak time in DA 3.0 ERG correlated with the GCLIPL thickness reduction $(\mathrm{r}=-0.341 ; p=0.005)$. The more significantly delayed b-wave peak time in LA 3.0 ERG was found to be associated with both global RNFL ( $\mathrm{r}=-0.367, p=0.001)$ and GCLIPL $(\mathrm{r}=-0.268 ; p=0.02)$ thicknesses (figure 1B).

Furthermore, GCL-IPL and RNFL thicknesses also demonstrated association with multiple other full-field ERG parameters, including both scotopic and photopic responses (table 2). GCL-IPL thickness correlated with the b-wave amplitude in rod response (DA 0.01 ERG) and in combined rod-cone responses (DA 3.0 and DA 12.0 ERGs). Similarly, global RNFL thickness was associated with the amplitude of b-wave in both rod (DA 0.01 ERG) and cone responses (LA 3.0 ERG).

The INL and ONL function also correlated with EDSS, lesion volume, and disease duration. Of interest, we found that MS disability and disease course parameters were also associated with full-field ERGs at baseline. There was a correlation between EDSS scores and the delayed b-wave peak time in cone response (figure $1 \mathrm{C}$ ); Total T2 lesion volume in the whole brain was also associated with multiple ERG parameters (table 2), including the b-wave peak times in DA 0.01, DA 12.0, and LA 3.0 ERGs (figure 1D), the a-wave peak times in DA 3.0 and LA 3.0 ERGs, and the implicit time of $30 \mathrm{~Hz}$ flicker ERG. In addition, there was a correlation observed between the disease duration and ERG parameters (table 2), including the b-wave peak time in DA 0.01 ERG, the a-wave peak time in DA 3.0 ERG, the a-wave and b-wave peak times in LA 3.0 ERG, and the implicit time and amplitude of $30 \mathrm{~Hz}$ flicker ERG.

Longitudinal study. Reduction in ERG amplitudes during the follow-up period. Fifty-two patients completed the 3year ERG follow-up. A reduction in full-field ERG amplitudes was observed over the follow-up period, 
A.a

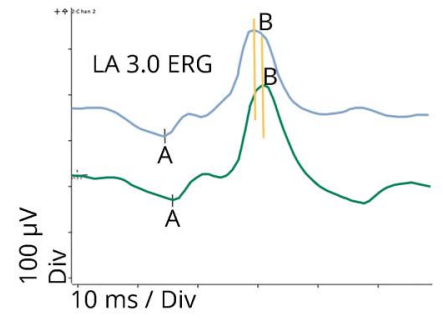

B.a

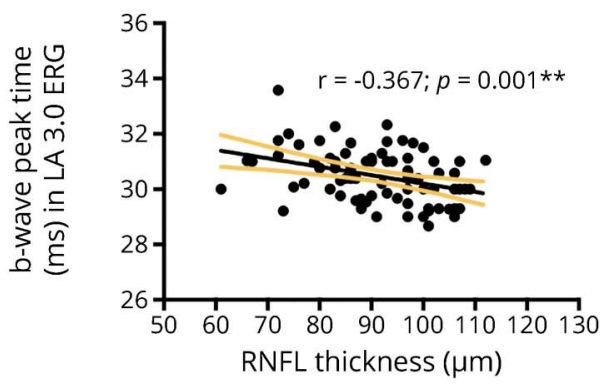

A.b

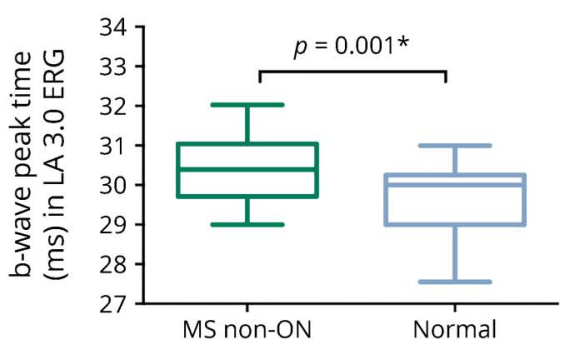

B.b

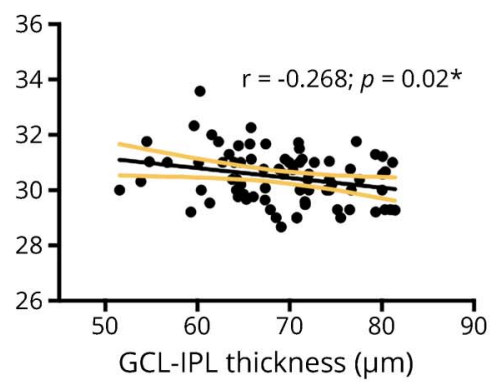

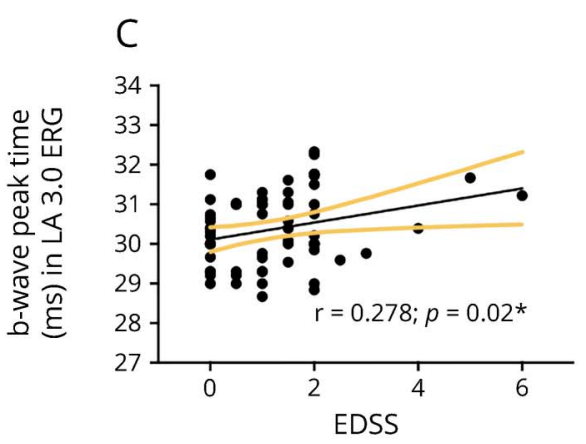
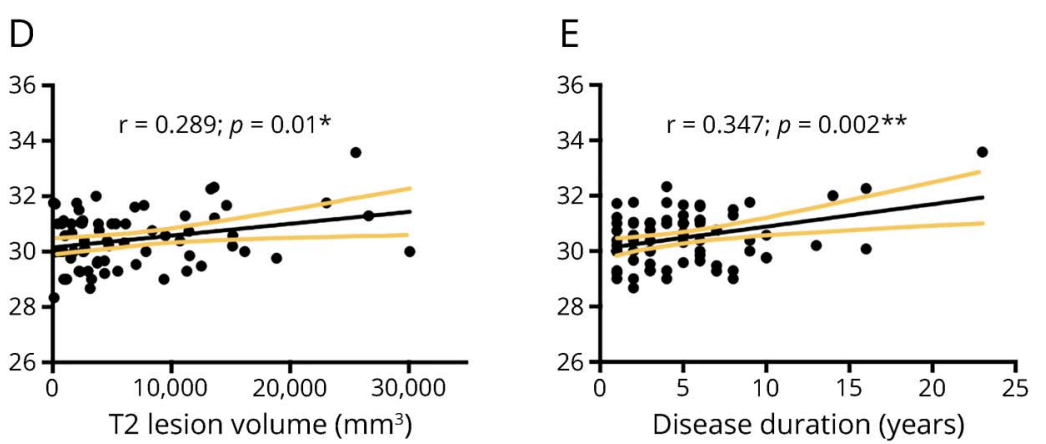

Peak time delay of the b-wave in light-adapted (LA) 3.0 electroretinography (ERG) and its association with retinal ganglion cell (RGC) loss and MS disease course. (A) Representative LA 3.0 ERG traces from a patient with MS (green) and a control (blue) (A.a); the $t$ test showed peak time delay in MS ( $n=77$ ) compared with controls $(n=30)$ (A.b). (B) Correlations between the delayed b-wave peak time in LA 3.0 ERG and the thicknesses of global retinal nerve fiber layer (RNFL) (B.a) and ganglion cell layer-inner plexiform layer (GCL-IPL) (B.b). (C-E) Correlations between the delayed b-wave peak time in LA 3.0 ERG and the Expanded Disability Status Scale (EDSS) (C), brain lesion volume on T2 MRI imaging (D), and disease duration (E).

including the b-wave amplitudes in DA 0.01 ERG $(p<0.001)$, DA 3.0 ERG $(p=0.002)$, and DA 12.0 ERG $(p=0.008)$, as well as the a-wave amplitudes in DA 3.0 ERG $(p=0.02)$, DA 12.0 ERG $(p=0.04)$, and LA 3.0 ERG $(p=0.005)$ (all with paired $t$ test and the ERG measurements are shown in table e-2 at http:// links.lww.com/A21). The most significant longitudinal amplitude loss was found in the b-wave of DA rod response (approximately $11 \%$ reduction, figure $2 \mathrm{~A}$ ). No changes were observed in ERG peak times. No ERG amplitude reduction was found in the controls $(p>$ 0.05 for all parameters, $n=12$ ) (figure $2 A$ ).

Correlation between ERG amplitude reduction and RNFL loss. Similar to our previous OCT study, ${ }^{19}$ we observed a significant reduction in global RNFL $(-1.73 \pm 2.61 \mu \mathrm{m})$ and GCL-IPL $(-1.10 \pm 1.66$ $\mu \mathrm{m})$ thickness in the study cohort over the follow-up period. Of interest, we found that the significantly reduced b-wave amplitude in DA 0.01 ERG was associated with the progressive RNFL loss $(r=0.424$, $p=0.001$, figure $2 \mathrm{~B}$ ). The correlation between ERG amplitude loss and RNFL reduction survived a multiple comparison analysis $(p=0.02)$, including lesion volume, EDSS, and lateral ventricle volume changes as other independent variables. Longitudinal change in GCL-IPL thickness did not significantly correlate with any of the ERG parameters.

During the 3-year follow-up period, 25 patients with MS developed new lesions in the brain, while the other 27 patients exhibited a relatively stable disease course, with no new brain lesions detected on annual MRI scans. However, both patient groups showed a similar trend in b-wave amplitude reduction in DA 0.01 ERG (figure 2C). Brain 


\begin{tabular}{|c|c|c|c|c|c|}
\hline \multicolumn{6}{|c|}{ Correlation between ERG, OCT, and disease course/disability parameters } \\
\hline ERG parameters & RNFL & GCL-IPL & EDSS & T2 Lesion & Duration \\
\hline \multicolumn{6}{|l|}{$0.01 \mathrm{DA}$} \\
\hline b-wave amplitude & $0.250(0.02)^{a}$ & $0.221(0.05)$ & - & - & - \\
\hline b-wave peak time & - & - & - & $0.278(0.02)$ & $0.259(0.02)$ \\
\hline \multicolumn{6}{|l|}{$3.0 \mathrm{DA}$} \\
\hline a-wave amplitude & - & - & - & - & - \\
\hline b-wave amplitude & $0.202(0.07)$ & $0.243(0.03)$ & - & - & - \\
\hline a-wave peak time & - & $-0.341(0.005)^{a}$ & - & $0.294(0.01)$ & $0.309(0.008)$ \\
\hline b-wave peak time & - & - & $-0.205(0.09)$ & - & - \\
\hline \multicolumn{6}{|l|}{$12.0 \mathrm{DA}$} \\
\hline a-wave amplitude & - & - & - & - & $0.189(0.10)$ \\
\hline b-wave amplitude & - & $0.232(0.04)$ & - & - & - \\
\hline a-wave peak time & - & - & - & - & $0.214(0.07)$ \\
\hline b-wave peak time & - & - & - & $0.334(0.005)^{a}$ & - \\
\hline \multicolumn{6}{|l|}{$3.0 \mathrm{LA}$} \\
\hline a-wave amplitude & - & $-0.183(0.10)$ & - & - & $0.188(0.10)$ \\
\hline b-wave amplitude & $0.244(0.03)$ & $0.329(0.003)^{a}$ & $-0.207(0.09)$ & $-0.204(0.08)$ & $-0.208(0.07)$ \\
\hline a-wave peak time & - & - & - & $0.312(0.008)$ & $0.268(0.02)$ \\
\hline b-wave peak time & $-0.367(0.001)$ & $-0.268(0.02)$ & $0.278(0.02)^{a}$ & $0.289(0.01)$ & $0.347(0.002)$ \\
\hline \multicolumn{6}{|l|}{$30 \mathrm{~Hz}$ Flicker } \\
\hline Amplitude & - & $0.200(0.08)$ & - & - & $-0.251(0.03)$ \\
\hline Implicit time & $-0.241(0.03)$ & - & - & $0.324(0.006)$ & $0.279(0.01)$ \\
\hline
\end{tabular}

Abbreviations: DA = dark adapted; EDSS = Expanded Disability Status Scale; ERG = electroretinography; GCL-IPL = ganglion cell layer-inner plexiform layer; $\mathrm{LA}=$ light adapted; OCT = optical coherence tomography; $\mathrm{RNFL}=$ retinal nerve fiber layer.

Data are $r$ value ( $p$ value). $p<0.05$ shown in bold; - : no correlation with $p>0.10$.

${ }^{\text {a }}$ Correlation survived multiple comparison analysis.

lesion volume changes were plotted against the ERG amplitude changes and no correlation was found $(p=0.24)$.

In addition, no correlation was found between the EDSS score changes and ERG amplitude reduction $(p=0.62)$. Brain atrophy and its association with the increase of LVV has been described in patients with MS. Here, we used the increased LVV as a measure of brain atrophy and again observed no correlation with ERG amplitude reduction $(p=0.50$, figure $2 \mathrm{D})$.

DISCUSSION Using comprehensive baseline and longitudinal full-field ERG evaluation, this study demonstrates evidence of functional alterations in the outer and middle retinal layers in non-ON eyes in patients with MS, which are to some degree associated with RNFL and RGC loss in the inner retina.

Progressive RNFL loss in non-ON eyes of patients with MS has been well described in previous OCT studies, ${ }^{4,11,21-25}$ and this study showed a comparable rate of continuous RNFL loss in non-ON eyes over the 3-year follow-up period. However, it is still unclear whether there are pathologic changes in the outer retinal layers. While loss of photoreceptors and bipolar cells have been demonstrated in postmortem studies of patients with MS, ${ }^{7}$ structural thinning in the INL in vivo has been observed only in some groups. Predominant macular thinning was demonstrated in a specific MS phenotype, ${ }^{9}$ while retinal layer changes in different MS types were investigated and INL thinning was identified to be associated with primary progressive MS ${ }^{11}$ Recently, ONL thickness in primary progressive MS was also found to be associated with the EDSS scores. ${ }^{26}$ Pathologic changes associated with the outer layers of the retina in MS could be subclinical or minor and therefore challenging to consistently detect on OCT. This may also explain why outer retinal atrophy in MS was more evident only in a postmortem study ${ }^{7}$ and widespread photoreceptor loss coupled with inflammatory changes was observed only in animal models. ${ }^{8,27}$ In addition, INL thickening was found in patients with MS with ON episodes, which was suggested to be linked with microcyst formation. ${ }^{28} \mathrm{~A}$ small degree 
A
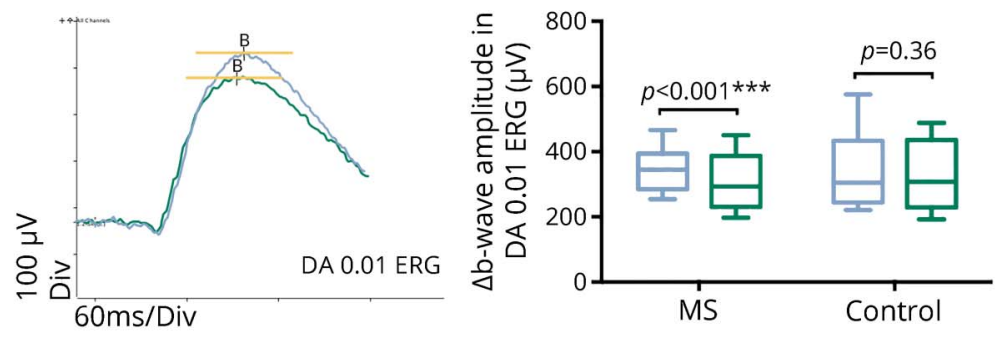

Baseline

Follow-up
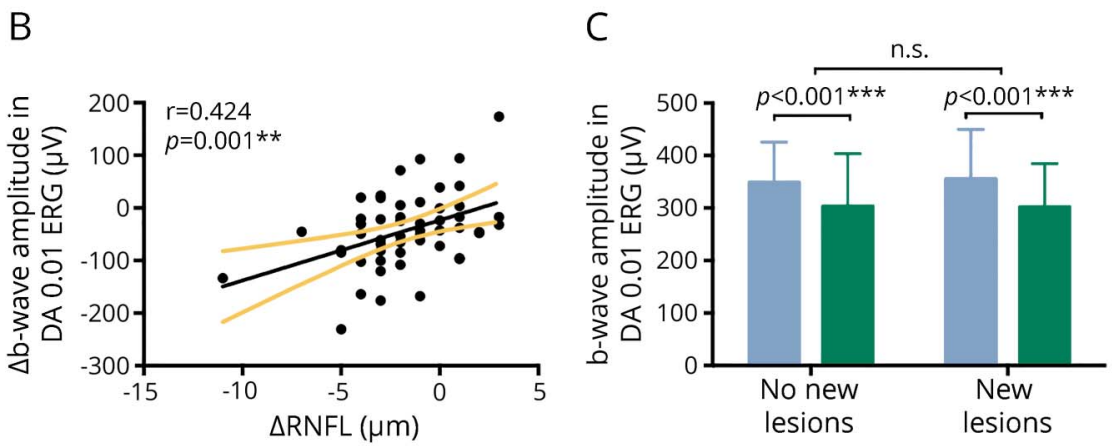

D

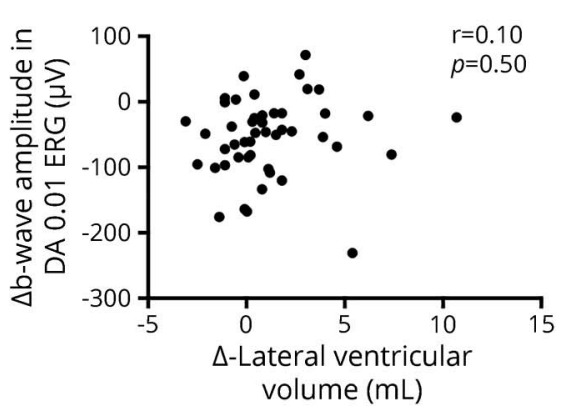

(A) Representative dark-adapted (DA) 0.01 electroretinography (ERG) traces from a patient with MS at baseline (blue) and year 3 (green). The paired $t$ test showed amplitude reduction in MS $(n=52)$ but not in the controls $(n=12)$. (B) There is a correlation between b-wave amplitude reduction in DA 0.01 ERG and retinal nerve fiber layer (RNFL) loss over the 3-year follow-up. (C) Patients $(n=25)$ who developed new brain lesions during the follow-up period and patients $(n=27)$ with no new lesions in the brain showed a similar trend of b-wave amplitude reduction. (D) No correlation was observed between brain atrophy, determined by lateral ventricular volume increase, and b-wave amplitude reduction.

of INL thickening was also found by meta-analysis. ${ }^{4}$ The effect of outer retinal layer thickening may potentially mask any underlying loss of thickness of the retinal layers in OCT studies.

The full-field ERG, on the other hand, provides a functional measure of the outer retina. ERG changes in patients with MS have been previously reported only in a limited number of cross-sectional studies. Although some retinal functional changes were described, ${ }^{13,15-17}$ most of the studies were not using the ISCEV standard and inconsistent results were shown. Retinal dysfunction was thought to be associated with $\mathrm{ON}$ attacks in earlier studies, ${ }^{15,16}$ but a more recent study using the ISCEV standard suggested no retinal dysfunction in non-MS patients with unilateral ON. ${ }^{14}$ In the current study, we did not observe any difference in the ERG recordings between $\mathrm{ON}$ and non-ON eyes. We found prolonged $\mathrm{a}$-wave and b-wave peak times of combined rod-cone response and LA cone response paradigms at baseline, suggesting that both rod- and cone-dominant pathways are affected in MS. The prolongation of ERG peak times may indicate subclinical retinopathy possibly related to neuroinflammatory changes. Retinal periphlebitis has been reported in patients with MS, which was thought to be related to MS severity. ${ }^{29,30}$ Although in the current study, we did not observe any active retinal periphlebitis during the follow-up period, it does not exclude some subclinical inflammatory changes in the retina, which might lead to functional changes in visual electrophysiology. Indeed, ERG anomalies have been widely used in clinical practice to assess various inflammatory/autoimmune retinal disorders, such as birdshot retinochoroidopathy, ${ }^{31}$ cancer-associated retinopathy, ${ }^{32}$ and multiple evanescent white dot syndrome. ${ }^{33}$ Localized inflammatory cellular infiltrates surrounding retinal veins have been observed in relapsingremitting and secondary progressive MS eyes. ${ }^{7}$ Antiretinal antibodies have also been reported in cellular proliferative disorders and in the pathogenesis of autoimmune retinopathy. ${ }^{32}$ Autoantibodies that are directed against neuronal antigens may also play a key role in axonal injury in $\mathrm{MS}^{13,34}$ and may partially be responsible for the subtle electrophysiology changes identified here. The association between ERG and RNFL measures indicated that the disease-related changes might affect the entire retina including both outer and inner retinal layers. At the baseline, a significant correlation was found between ERG parameters and MS disease duration, which suggested the presence of progressive retinal pathology throughout the entire disease course. The correlation between some of the ERG parameters and 
EDSS/T2 lesions can be related to the fact that patients with longer disease duration tend to have more severe clinical disability and brain lesion loads. ${ }^{35,36}$

In the longitudinal study, we also found a significant ERG amplitude reduction in non-ON eyes during the follow-up, suggesting progressive functional loss in the retina. Unlike ERG peak times, no statistically significant amplitude reduction was detected at baseline in comparison to controls (but there was an association between amplitude and RNFL), which may be due to high intersubject variability of the amplitude and the small sample size. ${ }^{37}$ In the follow-up study, by contrast, the absence of intersubject variability and the use of a paired $t$-test are likely to make the amplitude changes more evident.

Axonal damage has been identified in normalappearing white matter, which was not considered to be associated with demyelination throughout the CNS. ${ }^{38}$ However, the relationship between inflammation, de/remyelination, and neurodegenerative changes in MS remains poorly characterized. RNFL loss in MS was thought to be a result of either retrograde trans-synaptic degeneration or progressive primary RGC loss. ${ }^{4}$ Our previous study demonstrated significant association of RGC axonal and neuronal loss in non-ON eyes of patients with MS with both retinal dysfunction and postchiasmal damage of the visual pathway, indicating that RGC degeneration in MS might be a combined result of retrograde trans-synaptic degeneration originated in the postchiasmal part of the visual pathways and primary retinal pathology. ${ }^{6,39}$ In a separate publication, we also demonstrated the absence of significant loss of INL thickness in parts of the retina with long-standing and severe loss of RGCs caused by optic nerve diseases, ruling out retrograde trans-synaptic degeneration within the retina itself. ${ }^{40}$ Therefore, the current study provides an additional support to the idea of primary pathology affecting the entire thickness of the retina in MS by demonstrating a significant association between functional changes in the outer retina and RGC loss in the inner retina.

ERG changes were also associated with the disease duration and disability at baseline, but not with the disease activity during the follow-up. This result suggested that while the retinal pathology is progressing throughout the entire disease course, it is somewhat independent of the lesional disease activity in the brain.

One limitation of this study is that the significance of the correlations are mostly minor or borderline, which may be due to the fact that the pathologic changes in the outer retinal layers in MS are very subtle and therefore difficult to detect. This might also explain the lack of correlation in the longitudinal analysis between ERG changes and disability/brain atrophy. The absence of correlation between ERG and longitudinal GCL-IPL change can be potentially related to the fact that the full-field ERG is a measure of panretinal function, while GCL-IPL represents only the macula and perimacular regions. Future studies with larger sample size and longer follow-up are required to validate current findings.

To summarize, in this study we found subclinical deterioration in full-field ERGs in patients with MS, and both cone and rod pathways were involved. These previously unrecognized outer retinal functional changes are associated with RGC deficits and disease duration, suggesting that the subclinical retinal pathology in MS affects both the outer and inner retinal layers and may partially contribute to RGC loss in MS.

\section{AUTHOR CONTRIBUTIONS}

Yuyi You: drafting/revising the manuscript, analysis or interpretation of data, and obtaining funding. Elizabeth C. Graham and Ting Shen: analysis or interpretation of data. Con Yiannikas and John Parratt: study design and acquisition of data. Vivek Gupta: revising the manuscript. Joshua Barton: acquisition of data and analysis or interpretation of data. Michael Dwyer: analysis or interpretation of data. Michael H. Barnett: study design and acquisition of data. Clare L. Fraser: revising the manuscript and acquisition of data. Stuart L. Graham: revising the manuscript, study design, and study supervision. Alexander Klistorner: drafting/revising the manuscript, study design, analysis or interpretation of data, obtaining funding, and study supervision.

\section{STUDY FUNDING}

National MS Society (NMSS), National Health and Medical Research Council (NHMRC), Sydney Eye Hospital Foundation, and Sydney Medical School Foundation (SMSF).

\section{DISCLOSURE}

Y. You received research support from MHMRC, The Ophthalmic Research Institute of Australia, and Sydney Medical School. E.C. Graham and T. Shen report no disclosures. C. Yiannikas received travel funding from Genzyme and Ipsen and consulted for Allergan and Ipsen. J. Parratt served on the scientific advisory board of Genzyme, Novartis, and Biogen; received speaker honoraria from Biogen, Genzyme/Sanofi, and Merck Serono; received research support from Multiple Sclerosis Research Australia, Neil and Norma Hill Inaugural Junior Practitioner Fellowship. V. Gupta reports no disclosures. J. Barton received travel funding and/or speaker honoraria from Teva, Novartis, SanofiGenzyme, Merck, and Biogen. M. Dwyer served on the scientific advisory board of Novartis and EMD Serono; consulted for Claret Medical Inc; and received research support from Novartis. M.H. Barnett served on the scientific advisory board of Novartis and Genzyme-Sanofi; received travel funding from Teva; and received research support from Novartis, Genzyme-Sanofi, Bio-CSL, Biogen, NSW Government, the Nerve Research Foundation, and the University of Sydney. C.L. Fraser reports no disclosures. S.L. Graham served on the scientific advisory board of Allergan Australia; received travel funding and/or speaker honoraria from Allerga and Novartis; is a section editor of Clin Exp Ophthalmol; holds a patent for multifocal VEP electrode positioning and signal scaling; and received research support from Novartis, Allergan, and NHMRC. A. Klistorner holds patents for Electophysiological visual field measurement, Method and apparatus for objective electophysiological assessment of visual function, Stimulus method for multifocal visual evoked potential, Flexible electrode assembly and apparatus for measuring electrophysiological signals, and Flexible printed circuit board electrode 
assembly for measurement of electrophysiological signals and received research support from the Sydney University Foundation for Medical Research and the Sydney Eye Hospital Foundation. Go to Neurology. org/nn for full disclosure forms.

Received July 28, 2017. Accepted in final form October 9, 2017.

\section{REFERENCES}

1. Stys PK, Zamponi GW, van Minnen J, Geurts JJ. Will the real multiple sclerosis please stand up? Nat Rev Neurosci 2012;13:507-514.

2. Friese MA, Schattling B, Fugger L. Mechanisms of neurodegeneration and axonal dysfunction in multiple sclerosis. Nat Rev Neurol 2014;10:225-238.

3. Klistorner A, Arvind H, Nguyen T, et al. Axonal loss and myelin in early ON loss in postacute optic neuritis. Ann Neurol 2008;64:325-331.

4. Petzold A, Balcer LJ, Calabresi PA, et al. Retinal layer segmentation in multiple sclerosis: a systematic review and meta-analysis. Lancet Neurol 2017;16:797-812.

5. Martinez-Lapiscina EH, Arnow S, Wilson JA, et al. Retinal thickness measured with optical coherence tomography and risk of disability worsening in multiple sclerosis: a cohort study. Lancet Neurol 2016;15:574-584.

6. Klistorner A, Sriram P, Vootakuru N, et al. Axonal loss of retinal neurons in multiple sclerosis associated with optic radiation lesions. Neurology 2014;82:2165-2172.

7. Green AJ, McQuaid S, Hauser SL, Allen IV, Lyness R. Ocular pathology in multiple sclerosis: retinal atrophy and inflammation irrespective of disease duration. Brain 2010; 133:1591-1601.

8. Fairless R, Williams SK, Hoffmann DB, et al. Preclinical retinal neurodegeneration in a model of multiple sclerosis. J Neurosci 2012;32:5585-5597.

9. Saidha S, Syc SB, Ibrahim MA, et al. Primary retinal pathology in multiple sclerosis as detected by optical coherence tomography. Brain 2011;134:518-533.

10. Brandt $A U$, Oberwahrenbrock $T$, Ringelstein $M$, et al. Primary retinal pathology in multiple sclerosis as detected by optical coherence tomography. Brain 2011;134:e193; author reply e4.

11. Albrecht P, Ringelstein M, Muller AK, et al. Degeneration of retinal layers in multiple sclerosis subtypes quantified by optical coherence tomography. Mult Scler 2012;18:14221429.

12. Frishman LJ. Origins of the electroretinogram. In: Heckenlively JR, Arden GB, editors. Principles and Practice of Clinical Electrophysiology of Vision, 2nd ed. Cambridge, Massachusetts: MIT Press; 2006:139-183.

13. Forooghian F, Adamus G, Sproule M, Westall C, O'Connor $\mathrm{P}$. Enolase autoantibodies and retinal function in multiple sclerosis patients. Graefes Arch Clin Exp Ophthalmol 2007;245:1077-1084.

14. Fraser CL, Holder GE. Electroretinogram findings in unilateral optic neuritis. Doc Ophthalmol 2011;123:173178.

15. Papakostopoulos D, Fotiou F, Hart JC, Banerji NK. The electroretinogram in multiple sclerosis and demyelinating optic neuritis. Electroencephalogr Clin Neurophysiol 1989;74:1-10.

16. Coupland SG, Kirkham TH. Flash electroretinogram abnormalities in patients with clinically definite multiple sclerosis. Can J Neurol Sci 1982;9:325-330.

17. Pierelli F, Pozzessere G, Stefano E, Martelli M, Rizzo PA, Morocutti C. Pattern visual evoked potentials and flash electroretinogram in clinically definite multiple sclerosis. Eur Neurol 1985;24:324-329.

18. Marmor MF, Fulton AB, Holder GE, Miyake Y, Brigell M, Bach M. ISCEV standard for full-field clinical electroretinography (2008 update). Doc Ophthalmol 2009;118: 69-77.

19. Graham EC, You Y, Yiannikas C, et al. Progressive loss of retinal ganglion cells and axons in nonoptic neuritis eyes in multiple sclerosis: a longitudinal optical coherence tomography study. Invest Ophthalmol Vis Sci 2016;57:23112317.

20. Dwyer M, Silva D, Bergsland N, et al. Neurological software tool for REliable atrophy measurement (NeuroSTREAM) in multiple sclerosis. Neurology 2016;86: S45.005.

21. Fisher JB, Jacobs DA, Markowitz CE, et al. Relation of visual function to retinal nerve fiber layer thickness in multiple sclerosis. Ophthalmology 2006;113:324-332.

22. Talman LS, Bisker ER, Sackel DJ, et al. Longitudinal study of vision and retinal nerve fiber layer thickness in multiple sclerosis. Ann Neurol 2010;67:749-760.

23. Garcia-Martin E, Pueyo V, Almarcegui C, et al. Risk factors for progressive axonal degeneration of the retinal nerve fibre layer in multiple sclerosis patients. Br J Ophthalmol 2011;95:1577-1582.

24. Tatrai E, Simo M, Iljicsov A, Nemeth J, Debuc DC, Somfai GM. In vivo evaluation of retinal neurodegeneration in patients with multiple sclerosis. PLoS One 2012;7: e30922.

25. Narayanan D, Cheng H, Bonem KN, Saenz R, Tang RA, Frishman LJ. Tracking changes over time in retinal nerve fiber layer and ganglion cell-inner plexiform layer thickness in multiple sclerosis. Mult Scler 2014;20:1331-1341.

26. Behbehani R, Abu Al-Hassan A, Al-Salahat A, Sriraman D, Oakley JD, Alroughani R. Optical coherence tomography segmentation analysis in relapsing remitting versus progressive multiple sclerosis. PLoS One 2017;12: e0172120.

27. Chan CC, Caspi RR, Ni M, et al. Pathology of experimental autoimmune uveoretinitis in mice. J Autoimmun 1990;3:247-255.

28. Kaushik M, Wang CY, Barnett MH, et al. Inner nuclear layer thickening is inversley proportional to retinal ganglion cell loss in optic neuritis. PLoS One 2013;8:e78341.

29. Sequlcre J, Fernandez M, Salinas-Alaman A, Garcia-Layana A, Bejarano B, Villoslada P. Diagnostic accuracy of retinal abnormalities in predicting disease activity in MS. Neurology 2007;68:1488-1494.

30. Ortiz-Perez S, Martinez-Lapiscina E, Gabilondo I, et al. Retinal periphlebitis is associated with multiple sclerosis severity. Neurology 2013;81:877-881.

31. Holder GE, Robson AG, Pavesio C, Graham EM. Electrophysiological characterisation and monitoring in the management of birdshot chorioretinopathy. Br J Ophthalmol 2005;89:709-718.

32. Weleber RG, Watzke RC, Shults WT, et al. Clinical and electrophysiologic characterization of paraneoplastic and autoimmune retinopathies associated with antienolase antibodies. Am J Ophthalmol 2005;139:780-794.

33. Cheng JY, Luu CD, Yeo IY, Chee SP. The outer and inner retinal function in patients with multiple evanescent white dot syndrome. Clin Exp Ophthalmol 2009;37:478-484.

34. Vyshkina T, Kalman B. Autoantibodies and neurodegeneration in multiple sclerosis. Lab Invest 2008;88:796-807. 
35. Radue E-W, Barkhof F, Kappos L, et al. Correlation between brain volume loss and clinical and MRI outcomes in multiple sclerosis. Neurology 2015;84:1-10.

36. Manouchehrinia A, Westerlind H, Kingwell E, et al. Age related multiple sclerosis severity score: Disability ranked by age. Mult Scler 2017;23:1938-1946.

37. Hochstein GD, Molnar FE, Marmor MF. Intrasession variability of the full-field ERG. Doc Ophthalmol 2007; 115:77-83.

38. Evangelou N, Esiri MM, Smith S, Palace J, Matthews PM. Quantitative pathological evidence for axonal loss in normal appearing white matter in multiple sclerosis. Ann Neurol 2000;47:391-395.

39. Klistorner A, Garrick R, Barnett MH, et al. Axonal loss in non-optic neuritis eyes of patients with multiple sclerosis linked to delayed visual evoked potential. Neurology 2013; $80: 242-245$.

40. Sriram P, Graham SL, Wang C, Yiannikas C, Garrick $\mathrm{R}$, Klistorner A. Transsynaptic retinal degeneration in optic neuropathies: optical coherence tomography study. Invest Ophthalmol Vis Sci 2012;53: 1271-1275. 


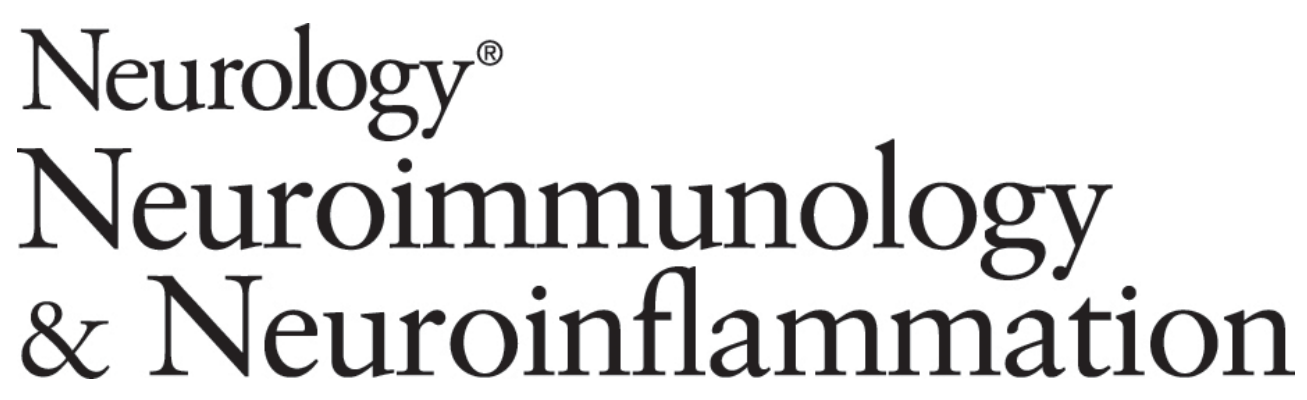

Progressive inner nuclear layer dysfunction in non-optic neuritis eyes in MS Yuyi You, Elizabeth C. Graham, Ting Shen, et al.

Neurol Neuroimmunol Neuroinflamm 2018;5;

DOI 10.1212/NXI.0000000000000427

This information is current as of December 15, 2017

Neurol Neuroimmunol Neuroinflamm is an official journal of the American Academy of Neurology.

Published since April 2014, it is an open-access, online-only, continuous publication journal. Copyright

Copyright $\odot 2017$ The Author(s). Published by Wolters Kluwer Health, Inc. on behalf of the American

Academy of Neurology.. All rights reserved. Online ISSN: 2332-7812.

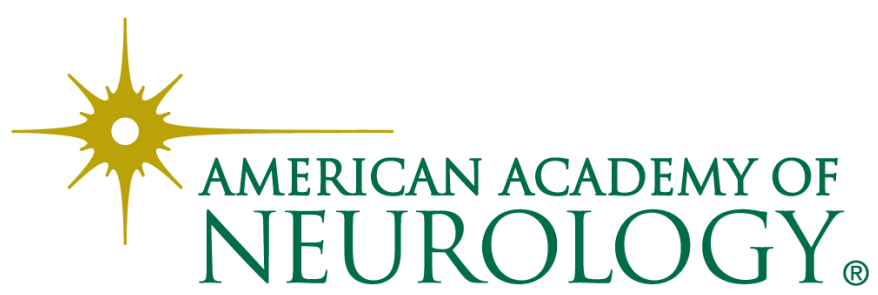




\section{Updated Information \& Services}

\section{Supplementary Material}

\section{References}

Citations

Subspecialty Collections

\section{Errata}

Permissions \& Licensing

\section{Reprints}

including high resolution figures, can be found at: http://nn.neurology.org/content/5/1/e427.full.html

Supplementary material can be found at: http://nn.neurology.org/content/suppl/2018/01/04/5.1.e427.DC1

This article cites 39 articles, 4 of which you can access for free at: http://nn.neurology.org/content/5/1/e427.full.html\#\#ref-list-1

This article has been cited by 1 HighWire-hosted articles: http://nn.neurology.org/content/5/1/e427.full.html\#\#otherarticles

This article, along with others on similar topics, appears in the following collection(s):

All clinical neurophysiology

http://nn.neurology.org//cgi/collection/all_clinical_neurophysiology

All Imaging

http://nn.neurology.org//cgi/collection/all_imaging

Multiple sclerosis

http://nn.neurology.org//cgi/collection/multiple_sclerosis

An erratum has been published regarding this article. Please see next page or:

/content/5/2/e444.full.pdf

Information about reproducing this article in parts (figures,tables) or in its entirety can be found online at:

http://nn.neurology.org/misc/about.xhtml\#permissions

Information about ordering reprints can be found online: http://nn.neurology.org/misc/addir.xhtml\#reprintsus

Neurol Neuroimmunol Neuroinflamm is an official journal of the American Academy of Neurology.

Published since April 2014, it is an open-access, online-only, continuous publication journal. Copyright

Copyright $\odot 2017$ The Author(s). Published by Wolters Kluwer Health, Inc. on behalf of the American Academy of Neurology.. All rights reserved. Online ISSN: 2332-7812.

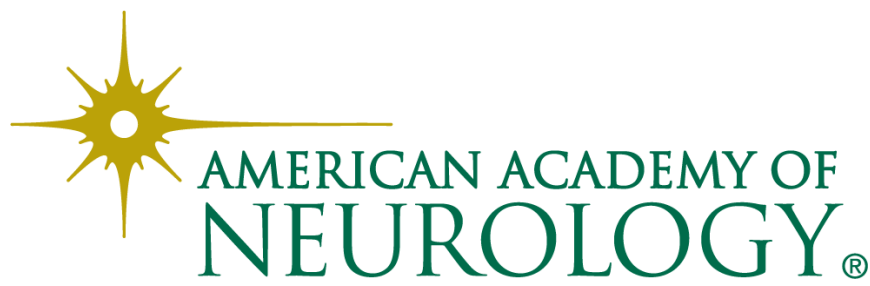




\section{CORRECTION}

\section{Progressive inner nuclear layer dysfunction in non-optic neuritis}

\section{eyes in MS}

Neurol Neuroimmunol Neuroinflamm 2018;5:e444. doi:10.1212/NXI.0000000000000444

In the Article "Progressive inner nuclear layer dysfunction in non-optic neuritis eyes in MS" by Y. You et al. ${ }^{1}$, there was an error in the last rows of Table e-1 and Table e-2, which should have listed "amplitude $(\mu \mathrm{V})$ " above "implicit time (ms)." The supplemental files were updated accordingly on January 10, 2018. Numerical values did not change. The authors regret the error.

\section{Reference}

1. You Y, Graham EC, Shen T, et al. Progressive inner nuclear layer dysfunction in non-optic neuritis eyes in MS. Neurol Neuroimmunol Neuroinflamm 2018;5:e427. 